\title{
Behavior of Hair Follicles in Vitiligo: Clinical Presentation and Discussion
}

\section{Sherif S Awad*}

Department of Dermatology and Venereology, Faculty of Medicine, Minia University, Egypt

\begin{abstract}
The color of the hair is dependent on the existence and function of the melanin producing cells, the melanocytes, in the hair matrix of the bulb. In vitiligo, the loss of color of the affected skin is due to loss of epidermal melanocytes, which may also be accompanied by loss of the bulbar melanocytes with subsequent loss of the hair color. Lymphocytic immune-cytotoxic process maybe involved in the destruction of epidermal or bulbar melanocytes. Hair depigmentation is said to follow surface depigmentation in long standing disease. This delay in development of leukotrichia in vitiligo could be claimed to the difference in melanization nature of hair follicle in comparison to epidermal melanization process. In rare cases, follicular melanocytes can be primarily destroyed in vitiligo without destruction of epidermal melanocytes. Apart from mature melanocytes, the hair follicles possess also immature form of melanocytes residing in the bulge area, outer root sheath, dermal papilla and sometimes the hair matrix. These immature cells usually escape the vitiligo immune destructive process.
\end{abstract}

The existence of leukotrichia used to be a sign of disease recalcitrance and expected treatment failure. In such cases several surgical procedures were introduced to help repigmenting the skin.

It was found that activation of the immature melanocytic precursors in hair follicles is responsible for the repigmentation of epidermis and hair follicles due to the supply with new mature melanocytes.

Keywords: Vitiligo; Hair follicle; Melanocytes; Stem cells

\section{Biology of Hair Coloring}

The hair bulb is the only site of pigment production for the hair shaft and contains highly melanogenic melanocytes and a minor subpopulation of poorly differentiated pigment cells [1]. In the adult hair follicle, pigmentation results from interactions between follicular melanocytes, matrix keratinocytes, and Dermal Papilla (DP) fibroblasts [2].

The "follicular-melanin unit" is residing in the "immune privileged" proximal hair bulb [3]. This follicular-melanin unit consists of one melanocyte for every five keratinocytes in the hair bulb as a whole; the ratio is $1: 1$ in the basal epithelial layer next to the dermal papilla [4]. Hair bulb melanogenic melanocytes differ from epidermal melanocytes in being larger, with longer and more extensive dendrites, producing two- to four fold larger melanosomes (Figure 1). Melanocytes of the hair bulb are known to have potential proliferation power and are not as indolent as epidermal melanocyte lacking completely this power of renewal. The hair bulb melanocyte system has been perceived as selfperpetuating whereby melanocytes involved in the pigmentation of one generation are also involved in the pigmentation of the next wave $[5,6]$. Follicular melanogenesis is characteristically cyclic in nature, as opposed to the continuous melanogenesis of epidermal pigmentation [7].

The increase in number of "white" hairs with or without vitiligo is time dependent due to extrinsic and intrinsic elements affecting the follicular melanocytes as well as epidermal melanocyte. Loss (mostly probably via apoptosis) may occur in both sun-exposed and covered skin with $10 \%$ reduction per decade after 30 years of age until 80 years, followed by more dramatic cell loss thereafter [8]. Again, oxidative stress can affect follicular melanocytes over time and may be a major factor in the loss of hair pigment, the reduction in overall numbers of melanocytes per follicle, and ultimately for induction of white hairs [9].

\section{Vitiligo and the Follicle}

In Vitiligo, destruction and loss of melanocytes from the skin epidermis is the cause of leukoderma. This destructive process may occasionally affect the active bulbar melanocytes too leading to the development of leukotrichia [5] (Figure 2). T-lymphocytes are well known pathological element during the development of vitiligo, expressing cytotoxic/apoptotic factors to induce melanocytic destruction [10,11]. These lymphocytes were also demonstrated perifollicularly in considerable density during disease activity in biopsies including black hairs within vitiligo patches. This density of perifollicular infiltrate was not found in cases with white hair follicles lacking the targeted antigen [12].

\section{Induction of Leukotrichia}

Distinct compartmentalization of skin melanocyte sub-populations

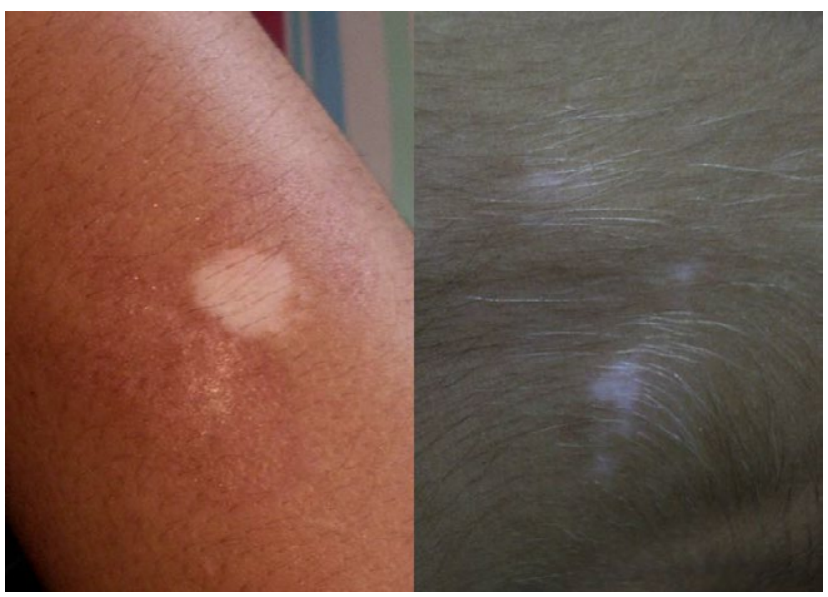

Figure 1: Hair within vitiliginous lesions may retain its color with normal appearance of follicles (Left panel) or could lose its color presenting as leukotrichia (Right panel).

Corresponding author: Sherif Awad, 8, Abdel Menam Garby Square, Minia Egypt, Tel : +201227340233; E-mail: sherifu@rocketmail.com

Received April 21, 2014; Accepted July 09, 2014; Published July 11, 2014

Citation: Awad SS (2014) Behavior of Hair Follicles in Vitiligo: Clinical Presentation and Discussion. Pigmentary Disorders 1:120. doi:10.4172/JPD.1000120

Copyright: (๑) 2014 Awad SS. This is an open-access article distributed under the terms of the Creative Commons Attribution License, which permits unrestricted use, distribution, and reproduction in any medium, provided the original author and source are credited. 


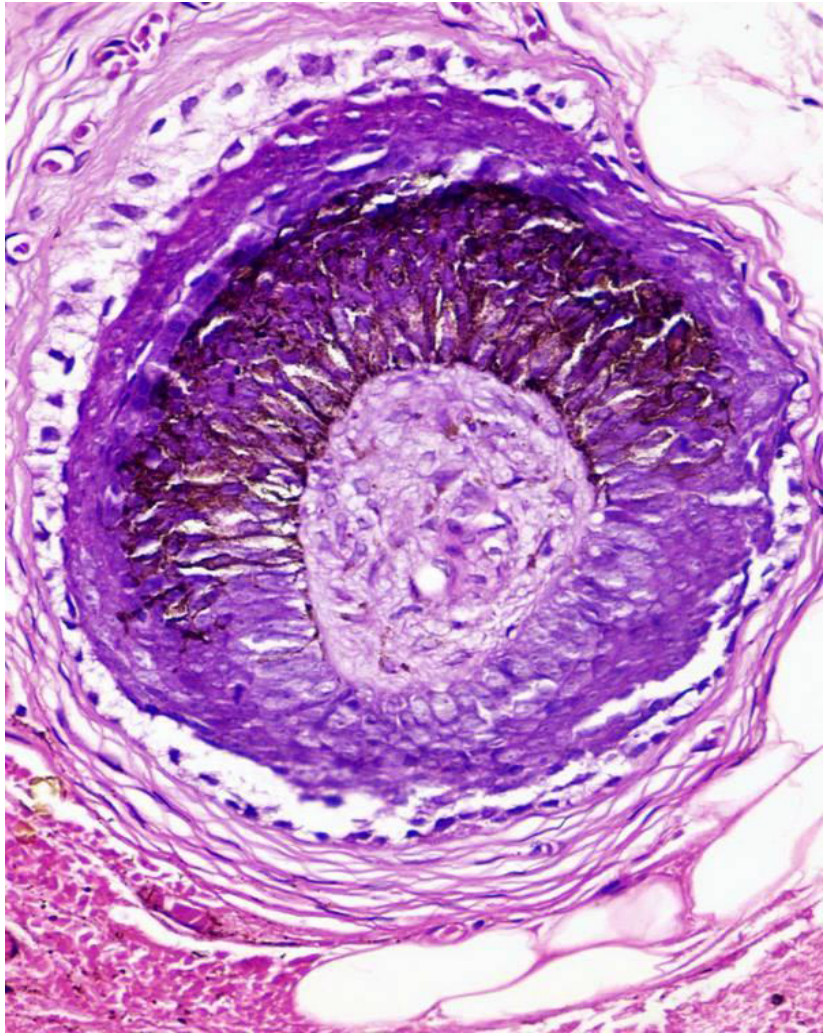

Figure 2: H\&E staining of normal hair follicle in cross section biopsy deep in the dermis showing hair bulb with central dermal papilla and hair matrix full with darkly stained follicular epithelium.

can be evidenced clinically by the selective or preferential targeting of epidermal melanocytes (but not follicular melanocytes) in vitiligo $[5,13]$.

Hair depigmentation usually follows surface depigmentation in long standing disease. Hair bulb melanocytes are said to be targeted first only in acute alopecia areata, whereas poliosis develops later in vitiliginous patches because of the loss of melanocytes from hair bulbs after the primary immunologic destruction of epidermal melanocytes [13].

This delay in vitiligo follicular depigmentation could be claimed to the stable condition of melanin contents in the follicles, whereas in the epidermis, the release of melanosomes from the melanocyte is followed by their transport to keratinocytes and subsequent exfoliation and biodegradation of melanin. The functioning melanocytes keep the equilibrium between release and exfoliation of melanin in normal situations. With the loss of melanocytes in the epidermis this equilibrium is lost too with subsequent biodegradation and exfoliation of melanin through epidermis. In hair follicles, melanin does not exfoliate or biodegrade as fast as within the epidermis. While melanin degrades almost completely in the differentiating layers of the epidermis, eumelanin granules transferred into hair cortical keratinocytes remain minimally digested [5].

Again, being within immune privileged hair bulb, the follicularmelanin unit can escape the autoimmune cytotoxic process which destroyed other epidermal melanocytes.

All these points may explain why hair can remain pigmented even during vitiligo activity with the considerable lymphocytic dermal or perifollicular infiltration.
Long standing disease duration is not the trigger for induction of poliosis as the development of follicular depigmentation is not a must sequence after certain duration of vitiligo. Neither the severity of disease extension nor duration of the vitiligo can be considered cause, as many vitiligo patients can retain the hair color even after many years of the disease (Figure 3).

Also, the hypothesis that hair color loss only follows the loss of skin pigment is not overall, although a common sequence, as some vitiligo patients can present with merely leukotrichia over normal looking pigmented skin surface with persistence of epidermal melanocytic function during the course of their disease (Figure 4). This means that mature bulbar melanocytes can be primarily destroyed in vitiligo without destruction of epidermal melanocytes.

Fully differentiated bulbar melanocytes undergo apoptosis during catagen [14], a similar process of destruction is expected during active immune process in vitiligo. Still, some less differentiated hair bulb melanocytes appear to survive catagen [7], and may possibly survive this immune/cytotoxic vitiliginous effects and be capable of differentiation and maintaining hair color within active vitiliginous patches.

\section{Follicular Melanocytes Reservoir}

The hair follicles contain several kinds of melanocytes, among them the non-mature amelanotic cells. Melanocyte stem cells survive catagen and are involved in restoration of the HF pigmentary unit after the HF enters into new anagen $[7,15,16]$.

The "re-differentiating" melanocytes of early anagen are likely newly recruited immature melanocytes derived from a melanocyte reservoir located in the upper, "permanent," outer root sheath. This view is supported by the observation that melanocyte stem cells located at the base of the permanent part of hair follicle are not only immature, but also slow cycling, self-maintaining and are fully competent to

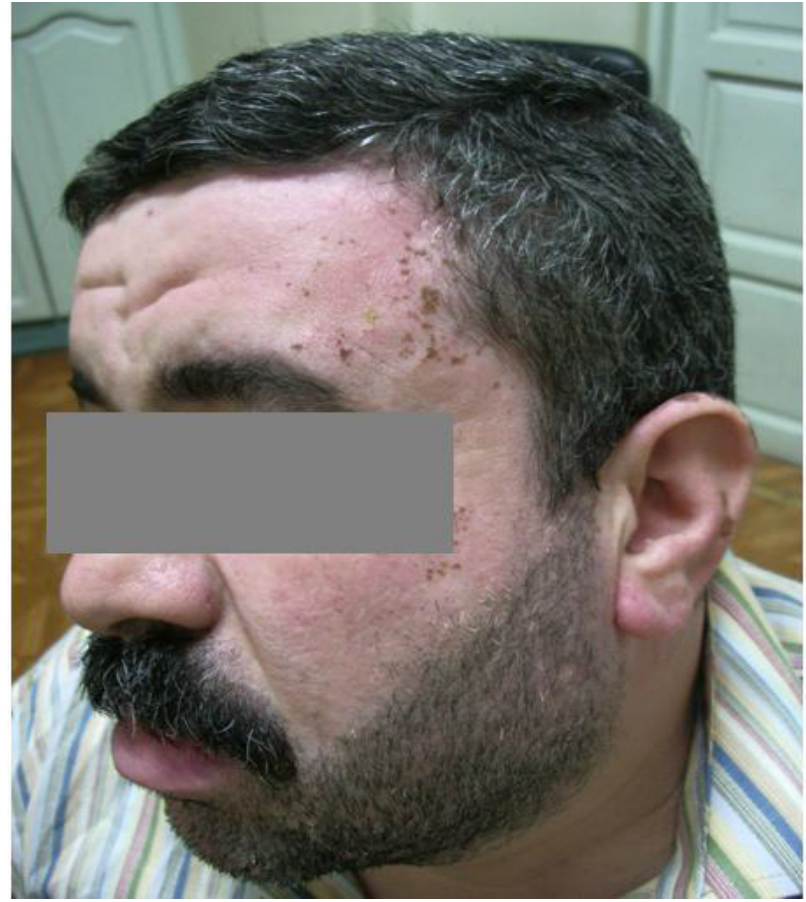

Figure 3: Patient with long standing vitiligo universalis (15 years vitiligo duration) with retained color of most of hair follicles on the scalp, beard, moustache and eye brows. 


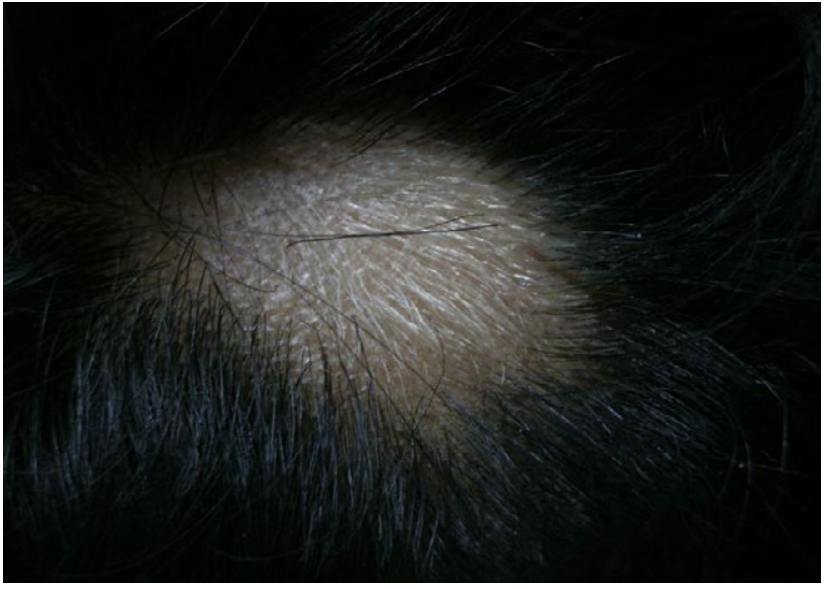

Figure 4: Scalp patch of Leukotrichia with complete loss of hair color presented over normally pigmented scalp in a vitiligo patient.

regenerating progeny at early anagen [17]. Sharov et al. stated that both bulge cells and follicular papilla cells may be the source of melanocytes during the new hair cycle as the original follicular melanocytes lose their functions during catagen [18]. The hair bulb may also possess a minor subpopulation of those poorly differentiated pigment cells [1].

\section{Melanocyte Related Proteins in Hair Follicle}

Several proteins and receptors were described and correlated to the melanogenesis process in the hair follicles. The famous c-Kit is required during the hair growth cycle for activation of melanocytes, although in stem cell compartment appears to exhibit stem cell factor SCF/c-Kit independence [15]. Some amelanotic dopa-negative melanocytes may also be distributed in the periphery of the bulb and the most proximal matrix $[1,19]$. All the dopa-positive cells, and also some dopa-negative melanocytes of the mid outer root sheath are (pre)melanosome gp100 positive [19]. Amelanotic hair follicle melanocytes are devoid of dopaoxidase activity, although low levels of the tyrosinase protein itself may be detected in some cells. Similarly, c-Kit and Bcl-2 reactive amelanotic melanocytes are present in this hair follicle compartment [20], but do not express the melanogenic enzymes TRP (Tyrosinase-Related Protein) 1 and TRP2 [19].

Yet, Slominski et al. stated that melanocytes localized to the hair follicle bulge (site of presumptive reservoir) express only TRP2, lacking TRP1, c-kit, and Ki67 immunoreactivities. Melanocytes from the fully developed anagen VI hair follicles express TRP2 only when located in the hair bulge, TRP2 and c-Kit only when located in the outer root sheath, and all three proteins, together with c-Kit, when located in the hair bulb matrix [21].

\section{Stimulation of Melanocyte Stem Cells}

New population of amelanotic spindle cells were clearly demonstrated in the infundibular and isthmus area of hair follicles in vitiliginous skin after ultraviolet radiation [22]. These cells were presumed stimulated melanocyte precursors and showed bipolar and tripolar morphology with minimal silver staining and absent Dopa reaction. These forms of follicular melanocytes were also described in culture by Kauser et al. stating that HFMs may show at least three distinct sub-populations, including highly pigmented dendritic bulbar melanocytes, less-differentiated tripolar cells, and an undifferentiated amelanotic bipolar sub-population [9]. This detected proliferation of melanocytic precursors preceded the characteristic perifollicular pigmentation, demonstrating the relevance of follicles during vitiligo repigmentation (Figure 5).

Such stimulation of melanocyte reservoir in the bulge areas was described before by Starrico who demonstrated amelanotic melanocytes in the ORS of hair follicles and proved that after phototherapy or mechanical removal of skin epidermis those immature cells start to proliferate, migrate and be stimulated [23-25].

\section{Hair Follicle and Vitiligo Regression}

The famous repigmentation pattern in vitiligo usually begins in the perifollicular area and treatment failure is expected in patches with leukotrichia. The presence of white hairs within vitiligo patches used to be a sign of recalcitrance and a poor prognostic signal [26,27].

The difficulty in pigmenting non-hairy glabrous skin such as on palms is well known, because of the absence of a melanocyte reservoir due to lack of hair follicles. In addition to hair bearing skin, with totally depigmented terminal hairs, which is less likely to respond due to the destruction of the melanocyte reservoir [28].

Again, this hypothesis is not a sharp indication for treatment failure as some vitiligo cases responded to phototherapy (Figure 6) in spite of the presence of poliosis, although others may fail to repigment.

\section{Surgical Procedures for Vitiligo}

The introduction of surgical procedures was mandated in such difficult to treat cases of vitiltigo with poliosis. Hair grafting was described to treat resistant areas aiming to provide new generation of melanocytes and precursors. Only the upper two thirds of the follicle was used and grafted when glaborous skin is targeted to avoid unwanted growth of coarse terminal hair. Successful surface repigmentation was attained after hair grafting [27].

Refilling of the melanocyte reservoir of the hair follicle by retrograde migration was described during treatment of patients with stable vitiligo lesions with depigmented hairs and expected depletion of the melanocyte reservoir in the hair follicle using transplantation of autologous cultured melanocytes in fibrin suspension [29].

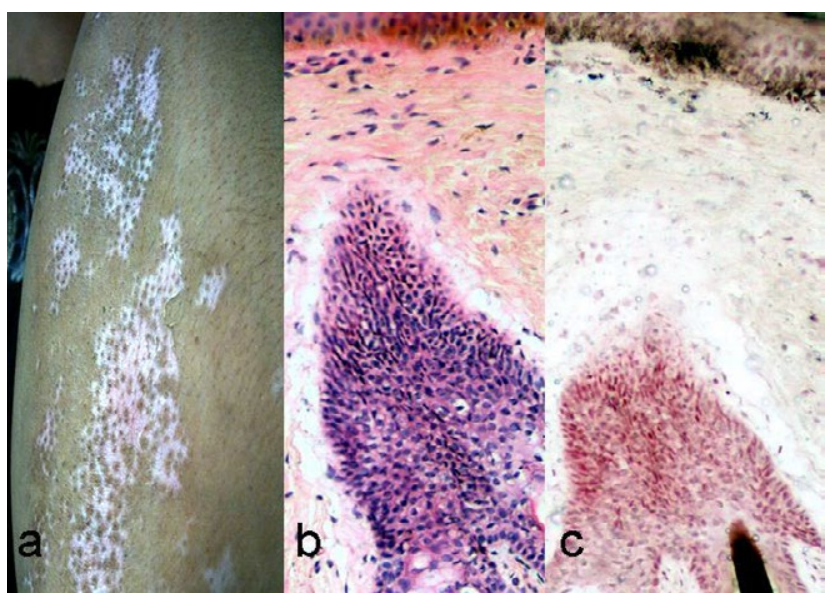

Figure 5: a) perifollicular pigmentation is a common pattern of recoloring in vitiligo skin after phototherapy. b) H\&E staining of biopsy including hair follicle within repigmenting spot revealed melanised surface epidermis with spindle and tripolar cells within follicular epithelium near infundibular and isthmus portion close to the bulge region. c) Fontana Masson Silver stain reveals darkly brown stained surface epidermis and mild red stained spindle and tripolar cells within the same follicular location. 


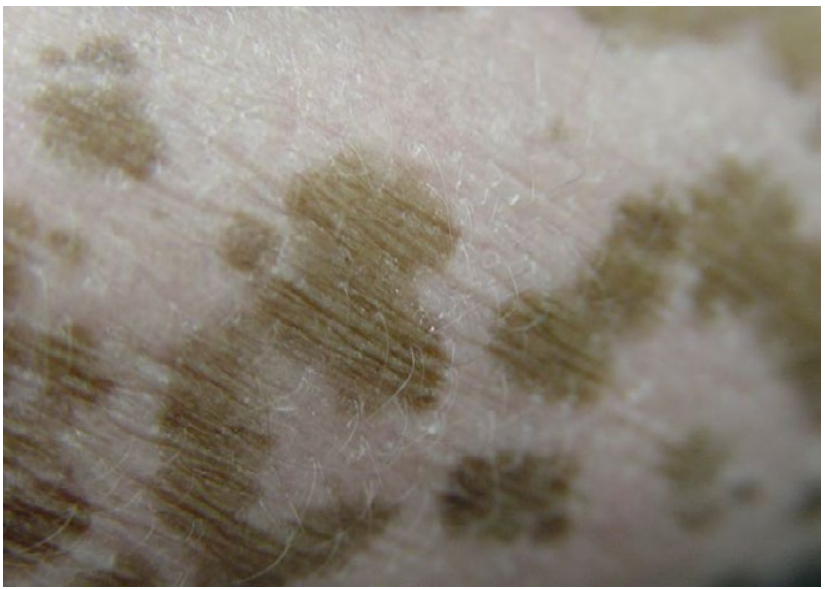

Figure 6: In spite of the loss of follicular color (leukotrichia), successful patchy repigmentation of vitiligo was obtained in this case after phototherapy.

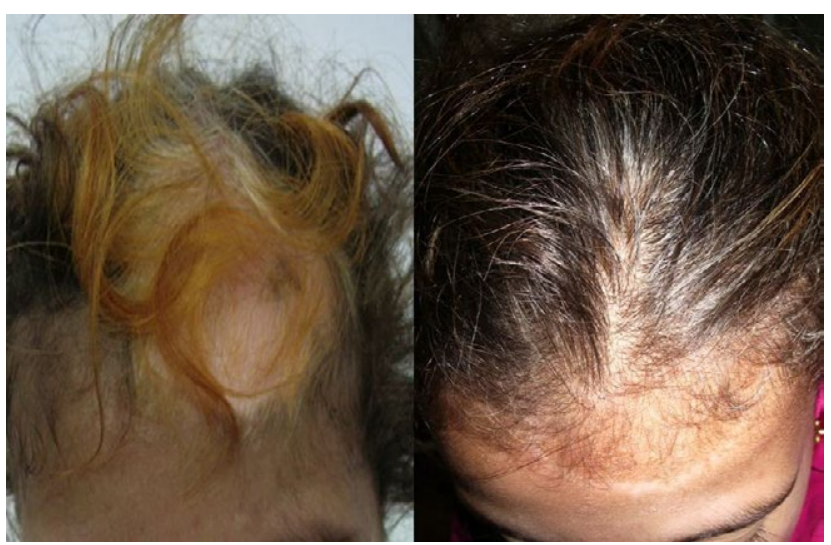

Figure 7: Child with vitiligo patch on forehead and scalp with manifested leukotrichia dyed with Henna as camouflage (Left Panel). After Epithelial grafting majority of hair follicles successfully repigmented following surface skin repigmentation and persisted for several years of follow up (Right Panel).

\section{Hair Repigmentation}

Interestingly, epithelial grafting on dermabraded vitiliginous skin with poliosis successfully repigmented the recalcitrant surface skin and also repigmented the white hair follicles afterward which persisted for several years (Figure 7). The duration required for hair follicles to repigment was not constant for all cases as was the surface skin but varied from 4 to 11 months after the procedure and this was claimed to different anagen durations in different sites of the body [23]. Retrograde migration theory was not the explanation here rather a stimulation of melanocyte reservoir in the bulge areas after dermabrasion and phototherapy following, in accordance to old previous reports demonstrating that mechanical removal of skin epidermis can stimulate those immature cells to start to proliferate and migrate [23-25].

Cells positively stained for c-kit were demonstrated in the outer root sheath of hair follicles after dermabrasion of vitiliginous skin in another study where dermabrasion proved to help repigmenting stable patches of vitiligo through stimulation of Melanocyte Stem Cells (MSCs) residing possibly also in hair follicles [30]. Although surface epidermal c-kit staining was not obtained yet staining of cells in the outer root sheath corresponded with the hypothesis that c-Kit is required mainly for hair supply rather than epidermal supply of MSCs [15,21,30]. Subsequently, repigmentation of leukotrichia in vitiligo patches after dermabrasion maybe expected too and should be examined.

Epilation of white hairs was advised in a study to accelerate the hair cycle, and new black hairs may emerge in new anagen phase [27].

\section{Conclusion}

Hair follicles in vitiligo my retain their normal color or lose it independently from disease duration, severity or extension. Poliosis is a possible manifestation in vitiligo after loss of functioning bulbar melanocytes and subsequent loss of melanin production and transfer to hair cortex/medulla. Several generations of melanocytes exist in the hair follicles, some are mature and functioning producing melanin and some are immature. The immature melanocytic cells may exist in 4 possible locations within the hair including; the bulge area, outer root sheath, dermal papillae, or within the hair bulb/matrix itself.

The destructive process during catagen stage or during vitiligo immune-cytotoxic activity affects only mature functioning cells, while other immature cells should escape this slaughter.

Stimulation of these follicular melanocytic precursors can lead to their maturation, differentiation and migration to supply new anagen follicles and surface epidermis with new functioning melanocytic population.

\section{References}

1. Tobin DJ, Bystryn JC (1996) Different populations of melanocytes are present in hair follicles and epidermis. Pigment Cell Res 9: 304-310.

2. Slominski A, Paus R (1993) Melanogenesis is coupled to murine anagen: toward new concepts for the role of melanocytes and the regulation of melanogenesis in hair growth. J Invest Dermatol 101: 90S-97S.

3. Christoph T, Müller-Röver S, Audring H, Tobin DJ, Hermes B, et al. (2000) The human hair follicle immune system: cellular composition and immune privilege. Br J Dermatol 142: 862-873.

4. Tobin DJ, Paus R (2001) Graying: gerontobiology of the hair follicle pigmentary unit. Exp Gerontol 36: 29-54.

5. Slominski A, Tobin DJ, Shibahara S, Wortsman J (2004) Melanin pigmentation in mammalian skin and its hormonal regulation. Physiol Rev 84: 1155-1228.

6. Sugiyama S (1979) Mode of redifferentiation and melanogenesis of melanocytes in mouse hair follicles. An ultrastructural and cytochemical study. J Ultrastruct Res 67: 40-54

7. Commo S, Bernard BA (2000) Melanocyte subpopulation turnover during the human hair cycle: an immunohistochemical study. Pigment Cell Res 13: 253-259.

8. Nordlund JJ (1989) The lives of pigment cells. Clin Geriatr Med 5: 91-108.

9. Kauser S, Westgate GE, Green MR, Tobin DJ (2011) Human hair follicle and epidermal melanocytes exhibit striking differences in their aging profile which involves catalase. J Invest Dermatol. 131: 979-982.

10. Saleh FY, Awad SS, Sadek IM (2013) Lymphocytic Expression of Fas and FasL Apoptotic Markers in Vitiligo, Clinical Medicine Research. 2: 105-109.

11. Lambe T, Leung JC, Bouriez-Jones T, Silver K, Makinen K, (2006) CD4 $T$ cell-dependent autoimmunity against a melanocyte neoantigen induces spontaneous vitiligo and depends upon Fas-Fas ligand interactions. J Immunol. 177: 3055-3062.

12. Anbar Tel-D, Abdel-Raouf H, Awad SS, Ragaie MH (2011) Perifollicular inflammatory infiltrate in vitiligo. Int J Dermatol 50: 234-237.

13. Tobin DJ (2008) Human hair pigmentation--biological aspects. Int $\mathrm{J}$ Cosmet Sci 30: 233-257.

14. Tobin DJ, Hagen E, Botchkarev VA, Paus R (1998) Do hair bulb melanocytes undergo apoptosis during hair follicle regression (catagen)? J Invest Dermato 111: 941-947. 
Citation: Awad SS (2014) Behavior of Hair Follicles in Vitiligo: Clinical Presentation and Discussion. Pigmentary Disorders 1:120. doi:10.4172/ JPD. 1000120

15. Botchkareva NV, Khlgatian M, Longley BJ, Botchkarev VA, Gilchrest BA (2001) $\mathrm{SCF} / \mathrm{c}$-kit signaling is required for cyclic regeneration of the hair pigmentation unit. FASEB J 15: 645-658.

16. Nishimura EK, Granter SR, Fisher DE (2005) Mechanisms of hair graying: incomplete melanocyte stem cell maintenance in the niche. Science 307: 720-724.

17. Nishimura EK, Jordan SA, Oshima H, Yoshida H, Osawa M, et al. (2002) Dominant role of the niche in melanocyte stem-cell fate determination. Nature 416: 854-860

18. Sharov A, Tobin DJ, Sharova TY, Atoyan R, Botchkarev VA (2005) Changes in different melanocyte populations during hair follicle involution (catagen). $\mathrm{J}$ Invest Dermatol 125: 1259-1267.

19. Horikawa T, Norris DA, Johnson TW, Zekman T, Dunscomb N, et al. (1996) DOPA-negative melanocytes in the outer root sheath of human hair follicles express premelanosomal antigens but not a melanosomal antigen or the melanosome-associated glycoproteins tyrosinase, TRP-1, and TRP-2. J Invest Dermatol 106: 28-35.

20. Grichnik JM, Crawford J, Jimenez F, Kurtzberg J, Buchanan M, et al. (1995) Human recombinant stem-cell factor induces melanocytic hyperplasia in susceptible patients. J Am Acad Dermatol 33: 577-583.

21. Slominski A, Wortsman J, Plonka PM, Schallreuter KU, Paus R, et al. (2005) Hair follicle pigmentation. J Invest Dermatol 124: 13-21.
22. Awad SS (2013) New population of amelanotic spindle cells are clearly demonstrated in vitiliginous skin after ultraviolet radiation. J Eur Acad Dermato Venereol .

23. Awad SS (2013). Repigmentation of poliosis after epithelial grafting for vitiligo Dermatol Surg. 39: 406-511.

24. STARICCO RG (1959) Amelanotic melanocytes in the outer sheath of the human hair follicle. J Invest Dermatol 33: 295-297.

25. Starrico RG (1959) Mechanism of migration of the melanocytes of the hair follicle into the epidermis following dermabrasion. J Invest Dermatol. 36: 99-104.

26. Seghal VN (1974) A clinical evaluation of 202 cases of vitiligo. Cutis. 14:439-46.

27. Na GY, Seo SK, Choi SK (1998) Single hair grafting for the treatment of vitiligo. J Am Acad Dermatol 38: 580-584.

28. Nordlund JJ, Halder RM, Grimes P (1993) Management of vitiligo. Dermatol Clin 11: 27-33

29. Hartmann A, Broecker EB, Hamm H (2008) Repigmentation of skin and hairs in stable vitiligo by transplantation of autologous melanocytes in fibrin suspension. J Eur Acad Dermatol Venereol 22: 624-626.

30. Awad SS (2012) Dermabrasion may repigment vitiligo through stimulation of melanocyte precursors and elimination of hyperkeratosis. J Cosmet Dermatol 11: 318-322. 